Jpn. J. Med. Sci. Biol., 51, Suppl. 1, S69-S80, 1998

\title{
Pathogenesis of Shigellosis: from Molecular and Cellular Biology of Epithelial Cell Invasion to Tissue Inflammation and Vaccine Development
}

\author{
Philippe J. SANSONETTI \\ Unité de Pathogénie Microbienne Moléculaire, INSERM U389, \\ Institut Pasteur, Paris, Cédex 15, France
}

\section{INTRODUCTION}

Shigellosis, or bacillary dysentery, is a bloody diarrhea caused by the invasion of the human colonic and rectal mucosa by Shigella, a gramnegative microorganism belonging to the family enterobacteriaceae. In the developing world, children are the major victims with 600,000 deaths every year. The symptoms are characterized by early watery diarrhea rapidly followed by fever, intestinal cramps, and emission of mucopurulent and bloody stools. Immediate complications may occur, such as hypoglykemia, seizure, toxic megacolon, and the hemolytic uremic syndrome (HUS). A chronic enteropathy may also be observed with delayed thriving of affected children. There are four species of Shigella (Shigella sonnei, Shigella flexneri, Shigella dysenteriae and Shigella boydii). The worldwide endemic form of the disease is caused by $S$. sonnei and $S$. flexneri. The epidemic form, which accounts for deadly outbreaks in developing areas, is caused by $S$. dysenteriae 1 . The latter, which was discovered in 1898 by the Japanese microbiologist Shiga, is still often referred to as the Shiga bacillus. It is characterized by the production of Shiga toxin, a potent cytotoxin accounting for the particular severity of the cases the Shiga bacillus usually causes. Shigella is a highly contagious microorganism which is transmitted directly from person to person by hand contact, or indirectly by contaminated food or water. High infectious capacity and rapid occurrence of multiple resistance to antibiotics make prevention and treatment of shigellosis a difficult task. No vaccine is yet available.

A key factor of the pathogenesis of shigellosis is the capacity of the causative microorganisms to invade epithelial cells (LaBrec, 1965). Based 
on in vitro assays, invasion encompasses three major steps: the capacity of bacteria to induce their own entry into epithelial cells by macropinocytosis (Clerc, 1987), their property to lyse the membrane of the endocytic vacuole and to escape into the cytoplasm (Sansonetti, 1986), their capacity to move intracellularly and to spread from cell to cell (Makino, 1986) by an actin-dependent process (Bernardini, 1989). This capacity of Shigella to interact with components of the eukaryotic cell, particularly its cytoskeleton, is a remarkable example of molecular cross-talk between a pathogen and its target cell.

\section{ENTRY OF SHIGELLA FLEXNERI INTO EPITHELIAL CELLS}

This phenotype is essential to the pathogenicity of $S$. flexneri. It is encoded by a $30 \mathrm{~kb}$ sequence (Maurelli, 1985) located on a $200 \mathrm{~kb}$ virulence plasmid (Sansonetti, 1982; Sansonetti, 1983). Two adjacent loci are necessary and sufficient to encode and secrete the Ipa proteins which elicit the formation of the entry focus, via localized actin polymerization (Clerc, 1987). One locus is composed of the ipa operon (invasion plasmid antigen) which encodes' four secreted proteins: IpaB (62 kDa), IpaC (42 $\mathrm{kDa})$, IpaD $(37 \mathrm{kDa})$ and IpaA $(70 \mathrm{kDa})$, which are the effectors of bacterial internalization (Ménard, 1993). In the bacterial cytoplasm, IpaB and $\mathrm{IpaC}$ are associated with a $18 \mathrm{kDa}$ chaperon, $\mathrm{IpgC}$, which prevents their aggregation and proteolytic degradation (Menard, 1994). Ipa proteins have no signal peptide; upon contact of the bacteria with the cell surface, they are secreted, already folded, from the cytoplasm to the extracellular milieu without a periplasmic phase. In the extracellular medium, IpaB and IpaC form a complex (Ménard, 1994). This property of secretion upon contact is based on the expression and assembly of a type III secretory apparatus composed of about 20 different proteins called Mxi-Spa (membrane expression of antigens and secretion of protein antigens) which bridge the inner and outer membrane. This secretory system is encoded by the $m x i$ and spa operons which are transcribed in opposite direction to the ipa operon. A complex formed by the association of IpaB and IpaD appears to regulate the flux of Ipa proteins through this apparatus (Ménard, 1994). Homologues of this system are shared by several other enteric pathogens such as Yersinia, Salmonella, and enteropathogenic Escherichia coli (EPEC). We have recently identified artificial compounds such as the Congo red which fully activate the Mxi-spa secretory system (Barahni, 1997).

Deletion of the ipa $\mathrm{B}, i p a \mathrm{C}$ and $i p a \mathrm{D}$ genes causes inactivation of the 
entry phenotype (Ménard, 1993). Mutation in the ipaA gene causes about 90\% decrease in entry efficiency (Tran Van Nhieu, 1997). Latex beads coated with the IpaB-C complex are internalized into HeLa cells (Menard, 1996). The entry foci elicited by IpaB-C-coated beads nevertheless cause limited cytoskeletal and membrane rearrangements, indicating that the actual entry of the bacteria is a more complex process. These experiments allow to propose that IpaB and IpaC constitute the primary effector of Shigella entry (Ménard, 1996). It is not yet definitely known whether these effectors bind surface receptors of the epithelial cell which may cause signals inducing the cytoskeletal rearrangements, or form a complex inserting into the epithelial cell membrane, thus inducing the cytoskeletal changes by a direct effect, but also by forming a protein translocator allowing the invading bacterium to inject other secreted proteins into the cytoplasmic compartment of the epithelial cell. Evidence indicates that the $\alpha 5 \beta 1$ integrin may be mediating interaction between a complex formed by the Ipa proteins and the target epithelial cell (Watarai, 1996). This would suggest a link between the Ipa proteins and the triggering of cytoskeletal rearrangements via a transmembrane receptor. We have also shown that IpaB could bind and recruit the hyaluronan-binding receptor CD44 on the epithelial cell surface. This is correlated with local recruitment and activation of ezrin, a protein involved in linking cytoskeletal components, including actin filaments, to the cell membrane, which also binds to the cytoplasmic domain of CD44 (Skoudy et al., manuscript submitted). The issue of membrane receptor activation therefore is not yet solved. The latter hypothesis which postulates insertion of the IpaB-C complex into the epithelial membrane itself is suggested by experiments showing that IpaC (De Geyter, 1997), as well as IpaB (Cabiaux et al., unpublished data) are able to cause lysis of calcein-loaded liposomes. Our recent data indicate that IpaA may be injected into the epithelial cell by such process in order to regulate the cytoskeletal rearrangements (Tran Van Nhieu, 1997). Constitution of a protein translocator remains to be definitely demonstrated in Shigella, and its possible function in causing direct polymerization of actin, thus by-passing receptor-mediated events, is currently studied. It is still possible that a combination of these two mechanisms is actually occuring.

The signals allowing actin nucleation-polymerization and cytoskeletal rearrangements are currently deciphered. Two major signalling pathways have been shown to be activated during Shigella entry. The protooncogene pp60 $0^{\text {c-src }}$ is recruited at the entry site and its activation allows the tyrosinephosphorylation of one of its major substrates, cortactin (Dehio, 1995). 
The actual role of cortactin in the cytoskeletal rearrangements is not yet defined. Small GTPases of the Rho family (i.e. RhoA, B and C) are also recruited at the entry site of Shigella. Their involvement has been demonstrated by treating cells prior to their infection by the C3 exoenzyme of Clostridium botulinum which inactivates Rho by ADPribosylation. This treatment decreases the rate of infection by $90 \%$ (Adam, 1996). These results have been confirmed (Watarai, 1997). The role of Rho proteins seems to be filament bundling and elongation in the filopodial extension of the entry focus, possibly in line with their physiological function in regulating stress cable formation in cells. Once elongated, filaments are tightly bundled by plastin (Adam, 1995). Several actin-associated proteins which constitute and regulate cell adhesion foci are present in association with the cytoskeletal rearrangements supporting bacterial entry (Ménard, 1996). This observation, in addition to the need for c-src and Rho, suggests that entry is similar to plaque formation. This led to study the function of vinculin in bacterial entry (Tran Van Nhieu, 1997). Shigella barely invades vinculin-deficient cells, and entry is restored by transfection with vinculin-expressing cDNA. In addition, the IpaA protein is also required for entry. Mutagenesis of the ipa $\mathrm{A}$ gene decreases entry by about $90 \%$. This protein, which appears to be injected while the bacterium is internalized, binds vinculin. This interaction allows activation of vinculin and its binding to cytoskeletal proteins which organize adhesion plaques, such as $\alpha$-actinin, thus constructing a stable structure allowing optimal entry into cells.

\section{ESCAPE OF S. FLEXNERI INTO THE CELL CYTOPLASM, INTRACELLULAR MOTILITY, AND CELL TO CELL SPREAD}

Once intracellular, S. flexneri rapidly lyse the membrane-bound vacuole and escape into the cytoplasm (Sansonetti, 1986). Ipa proteins account for this lysis (High, 1992). After escape from the vacuole, bacteria can move along the stress fibers that radiate from adhesion plaques to the nucleus (Vasselon, 1992). Bacteria also move in the cytoplasm by an actin-based movement which is caused by the formation of an F-actin comet tail at one pole of the bacteria (Bernardini, 1989). Bacterial movement inside the cytoplasm is random and rapid $(6-50 \mu \mathrm{m} / \mathrm{min})$. It occurs optimally at the stage of bacterial division (Goldberg, 1993). Observation of the comet tail in transmission electron microscopy after S1 myosin decoration of actin filaments shows short filaments with their "barbed" ends oriented towards the surface of the bacteria, indicating that addition of new actin monomers occurs at the bacterial surface (Egile and Sansonetti, unpublished 
results). These newly formed filaments are released and crosslinked into a comet-tail structure providing the motile force that achieves bacterial movement. The icsA (virG) gene, located on the large virulence plasmid, accounts for F-actin comet tail formation and movement (Bernardini, 1989). IcsA was discovered as a protein promoting tissue dissemination of Shigella, and was also named VirG (Makino, 1986). Virulence of a $\Delta i c s$ A mutant is strongly attenuated in vivo, thus making this mutation a basis for the development of live attenuated Shigella vaccine candidates (Sansonetti, 1991). IcsA is a $120 \mathrm{kDa}$ outer membrane protein. It is composed of a C-terminal transporter domain, IcsA $\beta$, allowing translocation and anchorage of the $\mathrm{N}$-terminal domain, IcsA $\alpha$, at the outer membrane (Suzuki, 1996). IcsA $\alpha$ accounts for actin polymerization. It shares no homology with any known protein, neither with cytoskeletal proteins, nor with ActA, its counterpart in L. monocytogenes (Domann, 1992; Kocks, 1992). On its N-terminus, IcsA $\alpha$ has a series of 6 repeats of 32 to 34 residues, very rich in glycin. IcsA is necessary and sufficient to induce actin polymerization and bacterial motility which can be reproduced with the same efficiency in cell-free extracts such as cytoplasmic extracts of Xenopus laevis eggs or human cells. Surface expression of IcsA into an $E$. coli $\mathrm{K} 12$ in these extracts causes actin polymerization and formation of actin comets correlated with motility of the bacterial bodies (Kocks, 1995; Golberg, 1995).

In wild type bacteria, IcsA is asymetrically distributed, since it is seen only at the bacterial pole opposite to the septation furrow in dividing bacteria. Inside infected cells, IcsA determines the site of actin assembly and the direction of movement. Two factors participate to polar localization: (i) a yet uncharacterized intrinsic property of IcsA may address the protein to the bacterial pole, although a significant proportion is still expressed over the entire cell surface; (ii) cleavage of $50 \%$ of the total amount of expressed IcsA occurs at the junction of its $\alpha$ and $\beta$ domains (Fukuda,1995) on a sequence - S756SRRASS762 - which is also a phosphorylation site for PKA (d'Hauteville, 1992). A bacterial surface protease, SopA, cleaves IcsA (Egile, 1997).

The mechanism of actin polymerization induced by IcsA requires the recruitment of a cytosolic protein complex in the bacterial vicinity which creates the proper conditions for actin polymerization. The following steps may be required: (i) actin nucleation; (ii) elongation of actin filaments; (iii) capping of the filaments pointed ends; (iv) bundling of the filaments. Several proteins have been detected in the Shigella actin tail such as $\alpha$ - 
actinin, plastin, VASP and vinculin. Recently, vinculin has been identified as the first ligand of IcsA (Suzuki, 1996). Vinculin has been shown to interact in vitro with IcsA by its globular head domain. Recruitement of vinculin at the pole of Shigella occurs before substantial accumulation of F-actin occurs. VASP, another focal adhesion protein is a ligand for ActA (Niebuhr, 1993) and for vinculin (Reinhard, 1996), suggesting that Listeria and Shigella have evolved similar motility mechanisms involving VASP and an ActA analog, vinculin.

When the moving organism reaches the inner face of the cytoplasmic membrane, a protrusion is formed which is absorbed by the adjacent cell (Prévost, 1992; Sansonetti, 1994). Interactions are established with components of the cellular junction which allow this passage into the adjacent cell. Expression of cadherins is required to allow absorbtion of these protrusions by adjacent cells (Sansonetti, 1994). Once in the adjacent cell, the bacteria are trapped inside a double-membrane bound pocket which is rapidly lysed, involving IcsB, a $57 \mathrm{kDa}$ protein which is encoded by a gene located upstream the ipa genes in the locus encoding the effectors of entry (Allaoui, 1992).

\section{APOPTOTIC KILLING OF MACROPHAGES BY S. FLEXNERI: ANOTHER EXPRESSION OF THE INVASIVE PHENOTYPE AND A LINK WITH INFLAMMATION}

In in vitro studies, invasive Shigella are engulfed by macrophages, but unlike their non-invasive mutants, they escape from the phagosome and rapidly induce apoptotic death of the macrophage (Zychlinsky, 1992). This observation has been confirmed in vivo, looking at infection of Peyer's patches in a rabbit ligated loop model of infection. Apoptosis is detected at the background level in areas where infection is carried out with a non invasive mutant, whereas numerous apoptotic cells can be seen if an invasive Shigella is used (Zychlinsky, 1996). IpaB is required for killing of macrophages. Direct cytotoxicity of IpaB has been demonstrated both by a genetic approach (Zychlinsky, 1994), and by microinjecting this purified protein into macrophages (Chen, 1996). The mode of action of IpaB appears to reside essentially in its capacity to bind the interleukin$1 \beta$ (IL-1 $\beta$ )-converting enzyme (ICE/Caspase 1), or a related protein (Chen, 1996). It is likely that the binding of IpaB on the ICE molecule somehow activates its proapoptotic potential as ICE emerges as an obligate intermediate for the signalling pathways that lead to programmed cell death. The fact that ICE mediates IpaB-induced macrophage death is 
confirmed by the observation that the YVAD-CHO tetrapeptide which inhibits cysteine proteases of the ICE family protects macrophages against Shigella-induced apoptosis (Chen, 1996).

If macrophages are pretreated with LPS, their activation leads to the expression of cytokines such as TNF $\alpha$, IL-6, and IL-1. However, IL-1 $\beta$ remains intracellular as a promolecule until these macrophages are infected by invasive Shigella and initiate their cell death program while releasing large quantities of this strongly pro-inflammatory cytokine under its mature $17 \mathrm{kDa}$ form (Zychlinsky, 1994).

\section{INFECTION OF THE INTESTINAL EPITHELIUM BY S. FLEXNERI: IN VIVO EXPRESSION OF THE INVASIVE PHENOTYPE}

Evidence obtained from experiments carried out in macaque monkeys and in the rabbit ligated intestinal loop model have indicated that Shigella primarily translocated through the epithelial lining via the $\mathbf{M}$ cells belonging to the FAE which covers the lymph nodes associated with the mucosa (Wassef, 1989; Sansonetti, 1991; Sansonetti, 1996). Once located in the dome region of the FAE, bacteria penetrate the macrophages which are particularly abundant. A significant proportion of these macrophages die, some of them showing typical aspect of apoptotic cells (Sansonetti, 1996; Zychlinsky, 1996). Our current hypothesis is that those macrophages, which are permanently exposed to bacterial material from the intestinal flora, are in a state of subactivation. In the presence of the cell death message that they receive from invading shigellae, these macrophages release massive amounts of IL-1 $\beta$ which initiate a strong inflammation representing the starting point of the disease (Zychlinsky, 1997). This inflammation may then spread beyond the lymphoid area. Efficient control of tissue inflammation and invasion by IL-1ra infused to rabbits during Shigella infection confirms the major role plaid by IL-1 in the pathogenesis of the disease (Sansonetti, 1995).

The initial inflammation increases the permeability of the epithelial barrier to Shigella. This has been modelled both in vitro and in vivo. Transmigration of polymorphonuclear leukocytes through the epithelium loosens the cellular junctions, thus facilitating Shigella entry (Perdomo, 1994; Perdomo, 1994). Inflammation can occur exclusively from the reaction elicited at the level of the infected lymph nodes, but it is also possible that the presence of Shigella on the apical side of the epithelium 
elicits a transepithelial signalling sufficient to induce local transmigration of PMN, thus causing the formation of multiple zones of bacterial invasion, far away from the lymph nodes (Beatty, 1997).

\section{CONCLUSION}

Thanks to a combination of in vitro and in vivo approaches, the pathogenesis of shigellosis is progressively unraveling some of its secrets. Several questions, however, remain open, such as: (i) understanding the colonic specificity of Shigella, (ii) understanding the high infectiosity of the pathogen, (iii) deciphering the signalling pathways that, upon expression of the invasive phenotype, lead either to entry in the presence of epithelial cells or to cell programmed death in the presence of macrophages, (iv) confirming that translocation of the epithelium occurs essentially via $\mathbf{M}$ cells, since inflammation occuring at distant sites may also facilitate entry, (v) identifying the signalling cascade which causes the severe inflammation observed during shigellosis, (vi) understanding the bases of immune protection against the disease.

The currently available data has led to the development of a series of promising live attenuated oral vaccine candidates against shigellosis. SC602, a double deletion mutant of $S$. flexneri 2a has been constructed in our laboratory. Its $\Delta i c s \mathrm{~A}$ (lack of intracellular motility and cell to cell spread) and $\Delta i u c$, iut (lack of expression of the iron chelator aerobactin and its membrane receptor) mutations reflect a rational approach for virulence attenuation. This vaccine candidate has recently been tested in human volunteers in the USA. It has proven safe and protective after a single oral dose since the vaccinees who were challenged with the wild type strain appeared fully protected (Koster $T$., et al., manuscript in preparation). A similar vaccine candidate has been constructed with $S$. dysenteriae 1 which will soon be also tested.

Shigellosis may hopefully become a paradigm of applying the results of an analytical approach of bacterial pathogenesis to the design of an efficient prevention strategy.

\section{ACKNOWLEDGMENTS}

I wish to thank all the members of Unite de Pathogénie Microbienne Moléculaire whose excellent work made this review possible. I am also very grateful to Colette Jacquemin for editing this manuscript. 


\section{REFERENCES}

1. Adam, T., Giry, M., Boquet, P. and Sansonetti, P.J. (1996): Rho-dependent membrane folding causes Shigella entry into epithelial cells. EMBO J 15: 3315-3321.

2. Adam, T., Arpin, M., Prévost, M.C., Gounon, P. and Sansonetti, P.J. (1995): Cytoskeletal rearrangements and the functional role of T-plastin during entry of Shigella flexneri into HeLa cells. J Cell Biol 129:367-381.

3. Allaoui, A., Mounier, J., Pré vost, M.C., Sansonetti, P.J. and Parsot, C. (1992): icsB: a Shigella flexneri virulence gene necessary for the lysis of protrusions during intercellular spread. Mol Microbiol 6:1605-1616.

4. Bahrani, F.K., Sansonetti, P.J. and Parsot, C. (1997): Secretion of Ipa proteins by Shigella flexneri: inducing molecules and kinetics of activation. Infect Immun $65:$ 4005-4010.

5. Beatty, W.L. and Sansonetti, P.J. (1997): Role of lipopolysaccharide in signaling to subepithelial polymorphonuclear leukocytes. Infect Immun $65: 4395$ 4404.

6. Bernardini, M.L., Mounier, J., d'Hauteville, H., Coquis-Rondon, M. and Sansonetti, P.J. (1989): Identification of ics A, a plasmid locus of Shigella flexneri which governs bacterial intra- and intercellular spread through interaction with F-actin. Proc Natl Acad Sci USA 86:3867-3871.

7. Chen, Y., Smith, M.R., Thirumalai, K. and Zychlinsky, A. (1996): A bacterial invasin induces macrophage apoptosis by binding directly to ICE. EMBO J 15 : 3853-3860.

8. Clerc, P. and Sansonetti, P.J. (1987): Entry of Shigella flexneri into HeLa cells: evidence for directed phagocytosis involving actin polymerization and myosin accumulation. Infect Immun $55: 2681-2688$.

9. Dehio, C., Prévost, M.C. and Sansonetti, P.J. (1995): Invasion of epithelial cells by Shigella flexneri induces tyrosine phosphorylation of cortactin by a pp60 c-src mediated signalling pathway. EMBO J $14: 2471-2482$.

10. De Geyter, C., Vogt, B., Benjelloun-Touimi, Z., Sansonetti, P.J., Ruysschaert, J-M., Parsot, C. and Cabiaux, V. (1997): Interaction of IpaC, a protein involved in entry of $S$. flexneri into epithelial cells, with lipid membranes. FEBS Letter $400: 149-154$.

11. Domann, E., Wehland, J., Rohde, M., Pistor, S., Hartl, M., Goebel, W., Leimeister-Wachter, M., Wuenscher, M. and Chakroborty, T. (1992): A novel bacterial virulence gene in Listeria monocytogenes required for host cell microfilament interaction with homology to the proline-rich region of vinculin. EMBO J $11: 1981-1990$.

12. Egile, C., d'Hauteville, H., Parsot, C. and Sansonetti, P.J. (1997): SopA, an outer membrane protease achieving secretion and polar localization of IcsA in S. flexneri. Mol Microbiol $23: 1063-1073$.

13. Francis, C.L., Ryan, T.A., Jones, B.D., Smith, S.J. and Falkow, S. (1993): Ruffles induced by Salmonella and other stimuli direct micropinocytosis of bacteria. Nature $364: 639-642$.

14. Fukuda, I., Suzuki, T., Munakata, H., Hayashi, N., Katayama, E., Yoshikawa, M. and Sasakawa, C. (1995): Cleavage of Shigella surface protein VirG 
occurs at a specific site, but the secretion is not essential for intracellular spreading. J Bacteriol 177 : 1719-1726.

15. Goldberg, M.B., Thériot, J.A. and Sansonetti, P.J. (1993): Regulation of surface presentation of IcsA, a Shigella protein essential to intracellular movement and spread, is growth phase dependent. Infect Immun 62:56645668.

16. Goldberg, M.B. and Thériot, J.A. (1995): Shigella flexneri surface protein IcsA is sufficient to direct actin-based motility. Proc Natl Acad Sci USA 92 : 6572-6576.

17. d'Hauteville, H. and Sansonetti, P.J. (1992): Phosphorylation of IcsA by cAMP-dependent protein kinase and its effect on intercellular spread of Shigella flexneri. Mol Microbiol $6: 833-841$.

18. High, N., Mounier, J., Prévost, M.C. and Sansonetti, P.J. (1992): IpaB of Shigella flexneri causes entry into epithelial cells and escape from the phagocytic vacuole. EMBO J $11: 1991-1999$.

19. Isberg, R. (1991): Discrimination between intracellular uptake and surface adhesion of bacterial pathogens. Science $252: 934-938$.

20. Kocks, C., Gouin, E., Tabouret, M., Berche, P., Ohayon, H. and Cossart, P. (1992): Listeria monocytogenes-induced actin assembly requires the actA gene product, a surface protein. Cell $68: 521-531$.

21. Kocks, C., Marchand, J.B., Gouin, E., d'Hauteville, H., Sansonetti, P.J., Carlier, M.F. and Cossart, P. (1995): The unrelated surface proteins ActA of Listeria monocytogenes and IcsA of Shigella flexneri are sufficient to confer actin-based motility on Listeria innocua and Escherichia coli respectively. Mol Microbiol 18 : 413-423.

22. LaBrec, E.H., Schneider, H., Magnani, T.J. and Formal, S.B. (1964): Epithelial cell penetration as an essential step in the pathogenesis of bacillary dysentery. J Bacteriol 88 : 1503-1518.

23. Makino, S., Sasakawa, C., Kamata, K., Kurata, T. and Yoshikawa, M. (1986): A virulence determinant required for continuous reinfection of adjacent cells on large plasmid in Shigella flexneri 2a. Cell $46: 551-555$.

24. Maurelli, A.T., Baudry, B., d'Hauteville, H., Hale, T.L. and Sansonetti, P.J. (1985): Cloning of plasmid DNA sequences involved in invasion of $\mathrm{HeLa}$ cells by Shigella flexneri. Infect Immun $49: 164-171$.

25. Ménard, R., Préost, M.C., Gounon, P., Sansonetti, P.J. and Dehio, C. (1996): The secreted Ipa complex of Shigella flexneri promotes entry into mammalian cells. Proc Natl Acad Sci USA 93 : 1254-1258.

26. Mé nard, R., Dehio, C. and Sansonetti, P.J. (1996): Bacterial entry into epithelial cells: the paradigm of Shigella. Trends in Microbiol $4: 220-226$.

27. Ménard, R., Sansonetti, P.J., Parsot, C. and Vasselon, T. (1994): Extracellular association and cytoplasmic partitioning of the IpaB and IpaC invasins of Shigella flexneri. Cell 79:515-525.

28. Menard, R., Sansonetti, P.J. and Parsot, C. (1994): The secretion of the Shigella flexneri Ipa invasins is induced by the epithelial cell and controlled by IpaB and IpaD. EMBO J $13: 5293-5302$.

29. Ménard, R., Sansonetti, P.J. and Parsot, C. (1993): Non polar mutagenesis of 
the ipa genes defines IpaB, IpaC and IpaD as effectors of Shigella flexneri entry into epithelial cells. J Bacteriol 175 : 5899-5906.

30. Mengaud, J., Ohayon, H., Gounon, P., Mége, R-M. and Cossart, P. (1996): E-cadherin is the receptor for internalin, a surface protein required for entry of Listeria monocytogenes into epithelial cells. Cell 84 : 923-932.

31. Niebuhr, K., Chakraborty, T., Rohde, M., Gazlig, T., Jansen, B., Kollner, P. and Wehland, J. (1993): Localization of the ActA polypeptide of Listeria monocytogenes in infected tissue culture cell lines: ActA is not associated with actin "comets". Infect Immun $61: 2793-2802$.

32. Perdomo, J.J., Gounon, P. and Sansonetti, P.J. (1994): Polymorphonuclear leukocyte transmigration promotes invasion of colonic epithelial monolayer by Shigella flexneri. J Clin Invest 93 : 633-643.

33. Perdomo, J.J., Cavaillon, J.M., Huerre, M., Ohayon, H., Gounon, P. and Sansonetti, P.J. (1994): Acute inflammation causes epithelial invasion and mucosal destruction in experimental shigellosis. J Exp Med 180 : 1307-1319.

34. Pré vost, M.C., Lesourd, M., Arpin, M., Vernel, F., Mounier, J., Hellio, R. and Sansonetti, P.J. (1992): Unipolar reorganization of F-actin layer at bacterial division and bundling of actin filaments by plastin correlate with movement of Shigella flexneri within HeLa cells. Infect Immun 60:40884099.

35. Reinhard, M., Rudiger, M., Jockusch, B.M. and Walter, U. (1996): VASP interaction with vinculin: a recurring theme of interactions with proline-rich motifs. FEBS Letters 399 : 103-107.

36. Sansonetti, P.J., Mounier, J., Prévost, M.C. and Mége, R.M. (1994): Cadherin expression is required for the spread of Shigella flexneri between epithelial cells. Cell $76: 829-839$.

37. Sansonetti, P.J., Kopecko, D.J. and Formal, S.B. (1982): Involvment of a large plasmid in the invasive ability of Shigella flexneri. Infect Immun 35 : 852-860.

38. Sansonetti, P.J., Hale, T.L., Dammin, G.J., Kapfer, C., Colins, H. and Formal, S. (1983): Alterations in the pathogenicity of Escherichia coli K-12 after transfer of plasmid and chromosomal genes from Shigella flexneri. Infect Immun $39: 1392-1402$.

39. Sansonetti, P.J., Ryter, A., Clerc, P., Maurelli, A.T. and Mounier, J. (1986): Multiplication of Shigella flexneri within HeLa cells: lysis of the phagocytic vacuole and plasmid-mediated contact hemolysis. Infect Immun 51:461-469.

40. Sansonetti, P.J., Arondel, J., Fontaine, A., d'Hauteville, H. and Bernardini, M.L. (1991): OmpB. (osmo-regulation): and ics A. (cell to cell spread): mutants of Shigella flexneri. Evaluation as vaccine candidates. Probes to study the pathogenesis of shigellosis. Vaccine 9:416-422.

41. Sansonettti, P.J., Arondel, J., Cavaillon, J.M. and Huerre, M. (1995): Role of IL-1 in the pathogenesis of experimental shigellosis. J Clin Invest 96:884892.

42. Sansonetti, P.J., Arondel, J., Cantey, R.J., Prévost, M.C. and Huerre, M. (1996): Infection of rabbit Peyer's patches by Shigella flexneri: effect of adhesive or invasive bacterial phenotypes on follicular-associated epithelium. 
Infect Immun $64: 2752-2764$.

43. Suzuki, T., Shinsuke, S. and Sasakawa, C. (1996): Functional analysis of Shigella VirG domains essential for interaction with vinculin and actin-based motility. J Biol Chem $271: 21878-21885$.

44. Tran, Van, Nhieu, G., Ben, Ze'ev, A. and Sansonetti, P.J. (1997): Modulation of bacterial entry in epithelial cells by association between vinculin and the Shigella IpaA invasin. EMBO J $16: 2717-2729$.

45. Tran, Van, Nhieu, G. and Isberg, RR. (1993): Affinity and receptor density are primary determinants of $\beta 1$ chain integrin-mediated bacterial internalization. EMBO J 12 : 1887-1895.

46. Vasselon, T., Mounier, J., Hellio, R. and Sansonetti, P.J. (1992): Movement along actin filaments of the perijunctional area and de novo polymerization of cellular actin are required for Shigella flexneri colonization of epithelial Caco2 cell monolayers. Infect. Immun $60: 1031-1040$.

47. Wassef, J., Keren, D.F. and Mailloux, J.L. (1989): Role of M cells in initial bacterial uptake and in ulcer formation in the rabbit intestinal loop model in shigellosis. Infect Immun $57: 858-863$.

48. Watarai, M., Funato, S. and Sasakawa, C. (1996): Interaction of Ipa proteins of Shigella flexneri with alpha5beta1 integrin promotes entry of the bacteria into mammalian cells. J Exp Med 183 : 991-999.

49. Watarai, M., Kamata, Y., Kozaki, S. and Sasakawa, C. (1997): rho, a small GTP-binding protein, is essential for Shigella invasion of epithelial cells. J Exp Med $185:$ 281-292.

50. Zychlinsky, A., Prévost, M.C. and Sansonetti, P.J. (1992): Shigella flexneri induces apoptosis in infected macrophages. Nature $358: 167-169$.

51. Zychlinsky, A., Fitting, C., Cavaillon, J.M. and Sansonetti, P.J. (1994): Interleukin 1 is released by macrophages during apoptosis induced by Shigella flexneri. J Clin Invest $94: 1328-1332$.

52. Zychlinsky, A., Kenny, B., Ménard, R., Prévost, M.C., Holland, I.B. and Sansonetti, P.J. (1994): IpaB mediates macrophage apoptosis induced by Shigella flexneri. Mol Microbiol $11: 619-627$.

53. Zychlinsky, A., Thirumalai, K., Arondel, J., Cantey, J.R., Aliprantis, A.O., Sansonetti, P.J. (1996): In vivo apoptosis in Shigella flexneri infections. Infect Immun 64 : 5357-5365.

54. Zychlinsky, A. and Sansonetti, P.J. (1997): Apoptosis as a proinflammatory event: what can we learn from bacteria-induced cell death ? Trends in Microbiol 5 : 201-204. 